The following scientific article was officially published in the Proceedings of the IEEE International Symposium on Biomedical Imaging. This article's citation is as follows:

Dionne, Olivier, Kondo Claude Assi, Sebastien Grenier, Hubert Labelle, François Guibault, and Farida Cheriet. "Simulation of the postoperative trunk appearance in scoliosis surgery." In 9th IEEE International Symposium on Biomedical Imaging (ISBI), (2012): pp. 1208-1211.

doi: $\underline{10.1109 / \text { ISBI.2012.6235778 }}$

The manuscript, as accepted by the publisher, is reproduced in the following pages.

(C) 2012 IEEE. Personal use of this material is permitted. Permission from IEEE must be obtained for all other users, including reprinting/republishing this material for advertising or promotional purposes, creating new collective works for resale or redistribution to servers or lists, or reuse of any copyrighted components of this work in other works. 


\title{
SIMULATION OF THE POSTOPERATIVE TRUNK APPEARANCE IN SCOLIOSIS SURGERY
}

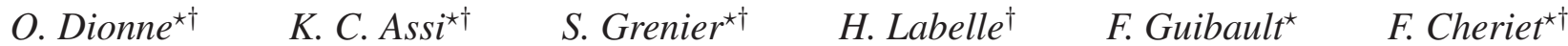 \\ * Department of Computer Engineering, École Polytechnique Montreal, \\ P.O. Box 6097, Succursale Centre-ville, Montréal, Québec, Canada H3C3A7 \\ $\dagger$ Sainte-Justine Hospital Research Center, 3175 Côte-Sainte-Catherine, \\ Montréal, Québec, Canada H3T1C5
}

\begin{abstract}
Persistence of external trunk asymmetry after scoliosis surgical treatment is frequent and difficult to predict by clinicians. This is a significant problem considering that correction of the apparent deformity is a major factor of satisfaction for the patients. A simulation of the correction on the external appearance would allow the clinician to illustrate to the patient the potential result of the surgery and would help in deciding on a surgical strategy that could most improve his/her appearance. We describe a method to predict the scoliotic trunk shape after a spine surgical intervention. The capability of our method was evaluated using real data of scoliotic patients. Results of the qualitative evaluation were very promising and a quantitative evaluation based on the comparison of the simulated and the actual postoperative trunk surface showed an adequate accuracy for clinical assessment. The required short simulation time also makes our approach an eligible candidate for a clinical environment demanding interactive simulations.
\end{abstract}

Index Terms - Scoliosis, biomedical modeling, soft tissue deformation, interactive surgical planning systems.

\section{INTRODUCTION}

Adolescent idiopathic scoliosis (AIS) is a complex threedimensional deformation of the trunk, with a prevalence of approximately $3 \%$ in the general population. Among patients with AIS, 9\% are treated by brace and one in a thousand needs surgery using posterior spinal instrumentation and fusion to correct the deformity. About 15,000 such surgeries are performed every year in North America and more than 4,000 were performed at the Sainte-Justine Hospital (SJH) in Montreal (Canada) during the last 30 years. The onset of the spinal deformation is variable and becomes more prominent during the adolescent growth spurt. The first manifestations are generally back pain and a visible rib hump.

Spinal correction has been improved over the years and

This research was funded by the Natural Sciences and Engineering Research Council of Canada. The equipment was financed by the Canadian Foundation for Innovation. Author 2 was also supported by Fondation SainteJustine and Fondation des Étoiles at Sainte-Justine Hospital of Montreal. has reached an adequate level. Unfortunately, this is not the case for the rib cage. Rib humps can persist after the surgery, which is a problem because the external appearance of the trunk is a major factor of satisfaction for the patient. The surgeon can decide to perform additional procedures but this decision is based only on his previous experience. A software system for the simulation of spinal surgery has already been developed [1], for the optimal planning of surgical procedures intended to correct scoliotic deformities. This tool, however, concerns only the internal spinal deformity and gives no information to estimate the effect of treatment on the patient's external appearance. Assessment by surgeons of scoliosis surgery outcomes are not significantly correlated with patient satisfaction and vary with the observer. A simulation of the correction, not only on the bone structures but also on the external appearance, would allow the clinician to illustrate to the patient the potential result of the surgery and would help in deciding on a surgical strategy that could most improve his/her appearance.

Physics-based deformable models have been used in biomedical applications, in particular for surgery simulation [2]. The two most popular approaches to physically modeling soft tissues are the Finite Element Method (FEM) [3] and Mass-Spring System (MSS) [4]. FEM still has a large memory footprint and computation times that limit interactive applications. MSS are less physically accurate than continuum mechanics models, and although they are simple in implementation, the specification of MSS parameters (spring constants, mesh topology) is not straightforward and remains a major difficulty of the approach.

We describe here a method for modeling the physical behaviour of the soft tissue of the trunk after spinal surgery to simulate the postoperative appearance of the trunk with adequate accuracy.

\section{MATERIALS AND METHODS}

\subsection{Data acquisition and $3 D$ reconstruction}

The surface geometry of the trunk is acquired using a calibrated system composed of four 3D optical digitizers (In- 
Speck Inc., Montreal, Canada), each assembled with a CCD camera and a structured light projector, placed around the patient. The acquisition process, for each scanner, consists in projecting and capturing four fringe patterns deformed by the trunk's external shape. The system then computes, by triangulation, the depth of each surface point relative to the reference plane of the digitizer. A fifth image, captured without fringes, defines the texture data mapped on the surface. The entire trunk geometry is obtained by registering and merging the partial surfaces obtained by each digitizer. This process takes 4-6 seconds with the patient standing still in the upright position, arms slightly abducted to prevent occluded areas in the field of view of the lateral scanners. The system demonstrated a reconstruction accuracy of $1.4 \mathrm{~mm}$ over the whole torso of a mannequin.

To reconstruct the bone structures of the trunk, a calibrated system based on biplanar radiographs, postero-anterior view (PA) and a lateral view (LAT), is employed. The 3D reconstruction of the spine from the standard PA and LAT views can be obtained using stereo-corresponding landmarks identified on both views. The accuracy of the $3 \mathrm{D}$ position of the identified landmarks was evaluated at $2.1 \pm 1.5 \mathrm{~mm}$. For the 3D surface reconstruction of the rib cage, our team has developed a novel method based only on two standard LAT and PA radiographs [5]. A detailed surface mesh of the patient's spine structures is then acquired by fitting a high-resolution atlas of 3D generic bone structures to the personalized data of the patient using dual kriging [6].

\subsection{Tetrahedral mesh generation of the trunk}

The procedure begins with the registered closed external surface of the torso and the surface mesh of the patient bone structures. As the latter may present self-intersection problems following the fitting procedure of the generic detailed model described in section 2.1, we extract a 2-manifold isocontour triangle mesh of the bone structures to best represent its initial shape. This is accomplished using the marching cube algorithm preceded by a discrete signed distance field evaluation of the initial reconstructed internal geometry. The void between the external trunk and bone surfaces is then filled using the tetrahedral mesh generator Tetgen [7]. A lateral cut of the resulting patient trunk mesh is shown in Fig. 1.

\subsection{Modeling the Scoliosis Surgical Procedure}

Let first consider the soft tissues of the human trunk as a homogeneous continuum occupying a domain $\Omega \subset \mathbb{R}^{3}$ connected to the bone structures $B \subset \mathbb{R}^{3}$. We denote the patient trunk, at equilibrium before surgery, as $V=B \cup \Omega$. Due to a surgical procedure, consisting in the reconfiguration of $B$ to $B^{\prime}$, an arbitrary point in the soft tissue initially at $\mathbf{x}=(x, y, z)^{T}$ is moved to position $\mathbf{x}^{\prime}=\left(x^{\prime}, y^{\prime}, z^{\prime}\right)^{T}$ inducing a change from $\Omega$ to $\Omega^{\prime}$ and the soft tissue exterior

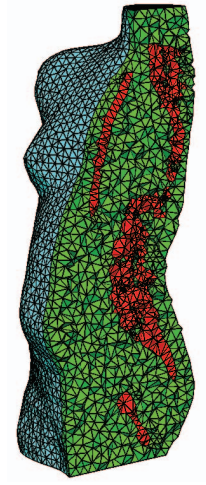

Fig. 1. Lateral cut of the tetrahedral patient trunk model

boundary surface of interest $S_{V}$ to $S_{V^{\prime}}$. The postoperative trunk appearance prediction problem can be expressed as follows : Given $B, B^{\prime}$ and $S_{V}$, find $S_{V^{\prime}}$.

Considering $\Omega$ as a deformable body with constraints given on its boundary by the prescribed displacements on $S_{B}$ (the surface of the bone structures), we formulate the trunk shape deformation model as a composition of two transformations (defined below):

$$
V^{\prime}=\tau \circ \mathcal{T}(V)
$$

where $\mathcal{T}$ is an initial approximation of the deformation field knowing the reconfiguration of the bone structures $S_{B}, S_{B^{\prime}}$ and $\tau$ is a relaxation transformation on $\Omega$ acting as a correction to $\mathcal{T}$.

\subsubsection{Transformation $\mathcal{T}$}

Let $L^{0}=\left\{\mathbf{l}_{i}^{0} \in \mathbb{R}^{3}, i=1, \ldots, n\right\}$ and $L^{1}=\left\{\mathbf{1}_{i}^{1} \in\right.$ $\left.\mathbb{R}^{3}, i=1, \ldots, n\right\}$ denote the pre- and postoperative sets of anatomical landmarks of the bone structures. The transformation $\mathcal{T}$ can be defined as a smooth interpolation function $F(\mathbf{x})=\left(f_{x}(\mathbf{x}), f_{y}(\mathbf{x}), f_{z}(\mathbf{x})\right)$ with constraints $F\left(\mathbf{l}_{i}^{0}\right)=$ $\mathbf{l}_{i}^{1},(i=1, \ldots, n)$. Thin plate splines are considered for the scalar interpolation functions $f_{x, y, z}: \mathbb{R}^{3} \rightarrow \mathbb{R}$.

\subsubsection{Transformation $\tau$}

According to energy principles in mechanics governing the static equilibrium of a deformable body, the solution sought is a configuration with minimal energy corresponding to the balance between the body's internal deformation energy and the work done by external forces. Considering a tetrahedral mesh of the trunk domain, let $E\left(\mathbf{X}_{b}, \mathbf{X}_{t}\right)$ be the total potential energy of the system for a given configuration where $\mathbf{X}_{t}=\left(\mathbf{x}_{1}^{(t)}, \ldots, \mathbf{x}_{m_{1}}^{(t)}\right)$ represents the coordinate vector of mesh vertices corresponding to the soft tissue and $\mathbf{X}_{b}=\left(\mathbf{x}_{1}^{(b)}, \ldots, \mathbf{x}_{m_{2}}^{(b)}\right)$ the coordinate vector of vertices associated to the underlying bone surface. We define a transformation $\tau: \mathbb{R}^{3} \rightarrow \mathbb{R}^{3}$ that maps a starting configuration $\mathbf{X}_{t}$ 
to its respective static equilibrium configuration $\mathbf{X}_{t}^{e q}$ :

$$
\mathbf{X}_{t}^{e q}=\underset{\mathbf{X}_{t}}{\operatorname{argmin}} E\left(\mathbf{X}_{b}, \mathbf{X}_{t}\right)
$$

We adapt Teschner et al. formulation of the internal energy [8] based on a tetrahedral mesh to take into account the contribution of gravity. An iterative energy minimization method [9] is used to solve Eq.(2). Algorithm 1 summarizes our method to predict the postoperative trunk appearance of a scoliotic patient.

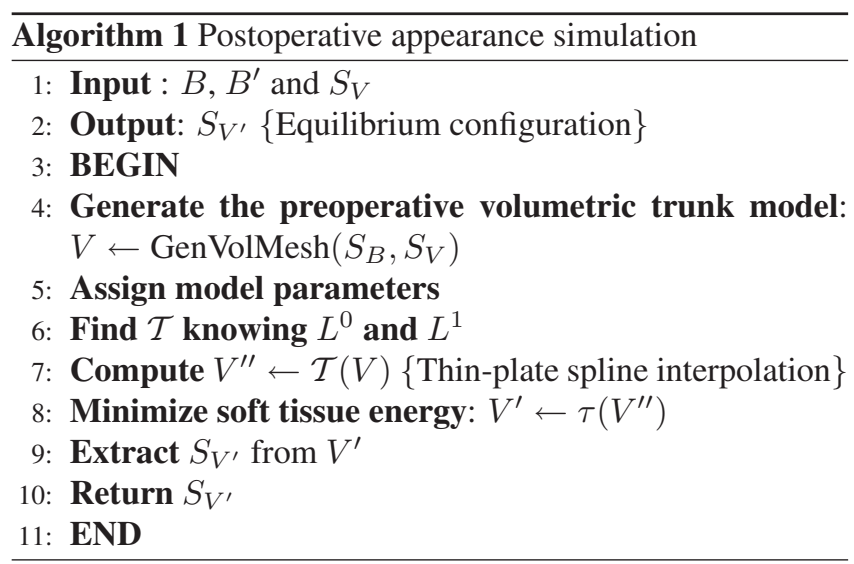

\subsection{Evaluation method}

The 3D visualization of the simulated and the actual postoperative external surface of the trunk allows a qualitative evaluation of the proposed method. For accuracy evaluation, we compared the predicted external surface to the actual postoperative external shape by computing the difference in maximal back surface rotation (BSR) of cross-sections between vertebral levels T3 (3rd thoracic vertebrae) and L5 (5th lumbar vertebrae), as presented in [10]. The BSR index is defined as the angle formed by the dual tangent to the posterior side of each section of the trunk surface and the axis passing through the patient's anterior superior iliac spines (ASIS), projected onto the axial plane. A difference less than $2.5^{\circ}$ for the BSR index (the smallest detectable difference for maximum BSR [11]) will be considered a clinically adequate accuracy.

\section{RESULTS AND DISCUSSION}

Simulation results for one patient are presented in Fig.2. The preoperative trunk shape shows a visible rib hump in the lumbar portion of the patient's back (Fig.2A). This prominence is also noticeable after applying transformation $\mathcal{T}$ on the overall patient geometry (Fig. 2B). However, it is considerably reduced following the minimization of the accumulated deformation energy induced in the soft tissue by $\mathcal{T}$ using transformation $\tau$ (Fig. 2C). The visual difference between the simulated and postoperative trunk surface (Fig. 2D) suggests a good approximation of the rib hump correction. On the other hand, dissimilarities are seen on the upper portion of the back.

Quantitative evaluation of the simulation was conducted

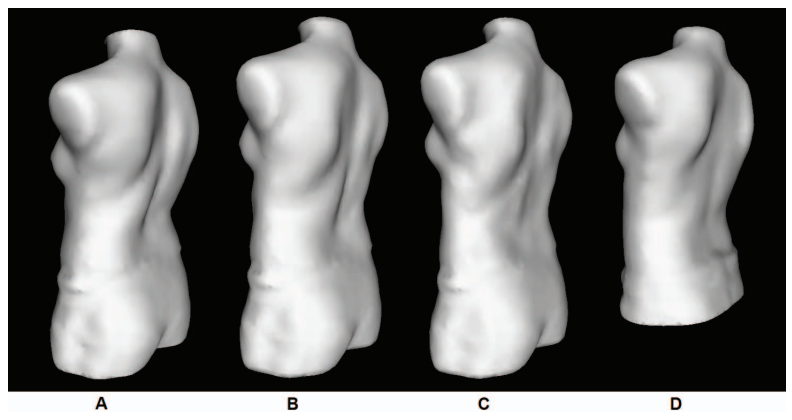

Fig. 2. Simulation results. (A) Preoperative initial patient trunk, (B) Trunk shape after geometric transformation $\mathcal{T}$, (C) Trunk shape after energy minimization transformation $\tau$ (D) Real postoperative patient trunk

using the BSR index computed from the predicted and acquired postoperative external geometries at various vertebral levels. A mean absolute difference of $1.15^{\circ}$ (thoracic) and $1.90^{\circ}$ (lumbar) on the BSR index were obtained. The evaluated difference on BSR index in the thoracic and lumbar regions fall in the range of the smallest statistically significant difference for the maximal back surface rotation.

We can observe certain discrepancies in the simulated trunk model compared to the actual result, notably in the upper left portion of the back (left scapula less prominent) and in the lumbar area (concavities on trunk surface which are simulation artifacts). Indeed, certain limitations of the current model prevent predicting the individual postoperative appearance of scoliotic patients. The first limit is that the geometry of the anatomical structures of the trunk is not completely personalized. The model of the spine is obtained from six landmarks per vertebra identified by an expert on a pair of radiographs. The surface model of the spine was generated by deforming generic vertebrae models obtained from serial CT-scans reconstruction of a dry specimen. Thus, the personalized information is limited to the $3 \mathrm{D}$ position of the identified anatomical landmarks which is not sufficient to describe the complex shape of each vertebra especially in presence of scoliosis pathology. In addition, these landmarks were manually identified and matched on a pair of views which is error-prone. Our team has recently developed a new method for the personalized $3 \mathrm{D}$ reconstruction of the scoliotic spine from hybrid statistical and image-based models [12]. This method will allow obtaining a refined and detailed geometry of the $3 \mathrm{D}$ vertebrae by an automatic segmentation of high level features such as the edges of vertebrae which are more visible from X-rays images. The surface model of the spine will be personalized and the computation of the surgical correction will be more accurate. Another limit related to the 
geometry is that the mesh generation was performed based only on the external surface of the trunk and the surface of the spine model and the rib cage. However, the soft tissue of the trunk is composed of three layers of tissue: skin, fat and muscles. To obtain a personalized tetrahedral mesh of each patient it will be useful to acquire MRI images of the trunk to extract the thickness of each layer. Additionally, the proposed model does not take into account the exact physical properties of the trunk soft tissue as the tissue elastic properties may differ depending on the type of scoliosis. Therefore, our team is currently developing a non invasive tool to assess the reducibility of the trunk from the acquisition of the external surface of the trunk in lateral bending position. Thus, some new constraints could be incorporated into the mathematical description to model the propagation of the spine correction through these personalized layers. We believe that, in order to enhance the accuracy of the simulation results, the rigidity constants should be calibrated from real data of a representative cohort of patients with different types of scoliosis.

All computations were performed on a standard personal computer equipped with a $2.8 \mathrm{GHz}$ Intel Core 2 Duo processor and $4 \mathrm{~GB}$ of RAM. The simulation time for a patient model composed of about $225 \mathrm{k}$ tetrahedra is approximately $1 \mathrm{~min}$ in a parallel running mode. This short simulation time appears to be an advantage for interactive scoliosis surgery planning systems and could be further optimized by transferring a portion of the computation to a graphics processing unit.

\section{CONCLUSION}

Our method allows an interactive simulation with an adequate accuracy which makes it very suitable for a clinical environment. This approach avoids the high FEM computation time required for a large number of tetrahedra. Furthermore, the accuracy of the simulation will be enhanced by calibrating the rigidity constants based on real patient data.

Once validated, the proposed simulator will benefit the surgeon by providing an enhanced preoperative planning tool and the patient by allowing her/him to readily understand the outcome of scoliosis surgery. Thus, this approach has the potential to lead to a non invasive predictive tool for the surgical treatment of scoliosis which will increase the quality of life of the patient by using non invasive imaging modalities.

In addition, the proposed approach will be adaptable to a broad range of applications, notably in the field of plastic surgery. The software development is modular and extensible and the system is designed to evolve and be adaptable to any region of the body for which data of external surface of the soft tissue and underlying bone structures are available.

\section{REFERENCES}

[1] C.-E. Aubin, H. Labelle, C. Chevrefils, G. Desroches, J. Clin, and A. B. Eng, "Preoperative planning simulator for spinal deformity surgeries," Spine, vol. 33, no. 20, pp. 2143-2152, 2008.

[2] U. Meier, O. López, C. Monserrat, M. C. Juan, and M. Alcaniz, "Real-time deformable models for surgery simulation: a survey," Computer Methods and Programs in Biomedicine, vol. 77, no. 3, pp. 183-197, 2005.

[3] R. M. Koch, M. H. Gross, F. R. Carls, D. F. von Büren, G. Fankhauser, and Y. I. H. Parish, "Simulating facial surgery using finite element models," in Proceedings of the 23rd Annual Conference on Computer Graphics and Interactive Techniques, 1996, pp. 421-428.

[4] D. Terzopoulos and K. Waters, "Physically-based facial modeling, analysis, and animation," Journal of $\mathrm{Vi}$ sualization and Computer Animation, vol. 1, no. 2, pp. 73-80, 1990.

[5] L. Seoud, F. Cheriet, H. Labelle, and J. Dansereau, "A novel method for the 3-D reconstruction of scoliotic ribs from frontal and lateral radiographs," IEEE Transactions in Biomedical Engineering, vol. 58, no. 5, 2011.

[6] S. Delorme, Y. Petit, J. A. de Guise, H. Labelle, C.-E. Aubin, and J. Dansereau, "Assessment of the 3D reconstruction and high-resolution geometrical modeling of the human skeletal trunk from 2D radiographic images," IEEE Transactions on Biomedical Engineering, vol. 50, no. 8, pp. 989-98, 2003.

[7] H. Si, "Tetgen: A quality tetrahedral mesh generator and three-dimensional Delaunay triangulator," http://tetgen.berlios.de/.

[8] M. Teschner, B. Heidelberger, M. Müller, and M. Gross, "A versatile and robust model for geometrically complex deformable solids," in Proceedings of the Computer Graphics International.

[9] O. Vinogradov, "A new method of molecular statics in polycrystals applications," Computational Materials Science, vol. 39, pp. 611-625, 2007.

[10] J. L. Jaremko, P. Poncet, J. Ronsky, J. Harder, and J. Dansereau, "Indices of torso asymmetry related to spinal deformity in scoliosis," Clinical Biomechanics, vol. 17 , no. 8, pp. 559-568, 2002.

[11] V. Pazos, F. Cheriet, J. Dansereau, J. Ronsky, R. F. Zernicke, and H. Labelle, "Reliability of trunk shape measurements based on 3-d surface reconstructions," European Spine Journal, vol. 16, no. 11, pp. 1882-1891, 2007.

[12] S. Kadoury, F. Cheriet, and H. Labelle, "Personalized Xray $3 \mathrm{D}$ reconstruction of the scoliotic spine from hybrid statistical and image-based models," Transactions on Medical Imaging, vol. 28, no. 9, pp. 1422-1435, 2009. 